\title{
Genotyping of Benzimidazole-Resistant and Dicarboximide-Resistant Mutations in Botrytis cinerea Using Real-Time Polymerase Chain Reaction Assays
}

\author{
Shinpei Banno, Fumiyasu Fukumori, Akihiko Ichiishi, Kiyotsugu Okada, \\ Hidetoshi Uekusa, Makoto Kimura, and Makoto Fujimura
}

First author: Plant Function Research Center, Toyo University, Itakura, Oura-gun, Gunma 374-0193, Japan; second, third, and seventh authors: Faculty of Life Sciences, Toyo University, Itakura, Oura-gun, Gunma 374-0193, Japan; fourth author: Agricultural, Food and Environmental Sciences Research Center of Osaka Prefecture, Shakudo, Habikino, Osaka, 583-0862, Japan; fifth author: Kanagawa Prefectural Agricultural Research Institute, Kamikisawa, Hiratsuka, Kanagawa 259-1204, Japan; and sixth author: Plant and Microbial Metabolic Engineering Research Unit, Discovery Research Institute (DRI), RIKEN, Hirosawa, Wako, Saitama 351-0198, Japan. Accepted for publication 3 November 2007.

\begin{abstract}
Banno, S., Fukumori, F., Ichiishi, A., Okada, K., Uekusa, H., Kimura, M., and Fujimura, M. 2008. Genotyping of benzimidazole-resistant and dicarboximide-resistant mutations in Botrytis cinerea using real-time polymerase chain reaction assays. Phytopathology 98:397-404.

Botrytis cinerea, an economically important gray mold pathogen, frequently exhibits multiple fungicide resistance. A fluorescence resonance energy transfer-based real-time polymerase chain reaction assay has been developed to detect benzimidazole- and dicarboximide-resistant mutations. Three benzimidazole-resistant mutations - ${ }^{198} \mathrm{Glu}$ to Ala (E198A), F200Y, and E198K - in $\beta$-tubulin BenA were detected using a single set of fluorescence-labeled sensor and anchor probes by melting curve analy-

assay resulted in the identification of a new benzimidazole-resistant BenA E198V mutation. This mutation conferred resistance to carbendazim as do E198A and E198K mutations. The isolates with BenA E198V mutation showed a negative cross-resistance to diethofencarb, but to a lesser extent than the E198A mutants. A survey of 210 B. cinerea field isolates revealed that most of benzimidazole-resistant isolates possessed the E198V or E198A mutation in the BenA gene, and the I365S mutation in the $B c O S 1$ gene was also frequently observed in Japanese isolates. However, benzimidazole-resistant isolates with BenA F200Y or E198K mutations, which confer the diethofencarb-insensitive phenotype, were rare. Our BenA and BcOS1 genotyping is a rapid and reliable method that is suitable for monitoring the fungicide-resistant field population.
\end{abstract} sis. Similarly, three dicarboximide-resistant mutations-I365S, V368F plus $\mathrm{Q} 369 \mathrm{H}$, and $\mathrm{Q} 369 \mathrm{P}$ - in the histidine kinase $\mathrm{BcOS1}$ were successfully distinguished. Unassigned melting profiles in BenA genotyping
Additional keywords: Botryotinia fuckeliana.
Botrytis cinerea (anamorph of Botryotinia fuckeliana (de Bary) Whetzel) causes gray mold on many economically important vegetable, fruit, and ornamental plants. The chemical control of gray mold in different crops has been adversely affected by the development of fungicide resistance in $B$. cinerea. Isolates of $B$. cinerea that are resistant to benzimidazole (e.g., carbendazim, benomyl, and thiophanate-methyl) and dicarboximide (e.g., iprodione and procymidone) fungicides are distributed worldwide $(3,15,28)$. Benzimidazoles inhibit the assembly of microtubules by binding to $\beta$-tubulin in fungi (6), and in most cases, mutations at amino acid positions 198 and 200 in the $\beta$-tubulin gene confer benzimidazole resistance in field isolates of many plant pathogens $(1,14,18,27)$. The $N$-phenylcarbamate diethofencarb is inactive against benzimidazole-sensitive strains but exhibits fungicidal activity against resistant strains with glutamic acid at position 198 to glycine (E198G) or E198A mutations in the $\beta$-tubulin gene by binding to the mutant $\beta$-tubulin $(7,8,11)$. Three types of benzimidazole-resistant strains of $B$. cinerea have been isolated from fields; mutations within the $\beta$-tubulin BenA gene of these isolates produced different phenotypes (27). The BenA E198A mutant strains were detected worldwide as a major benzimidazole-resistant strain in fields; they are highly resistant to the benzimidazole

Corresponding author: M. Fujimura; E-mail address: fujimura@itakura.toyo.ac.jp

doi:10.1094/PHYTO-98-4-0397

(C) 2008 The American Phytopathological Society carbendazim and sensitive to the negative cross-resistant fungicide diethofencarb. After the introduction of a mixture of diethofencarb and carbendazim (or thiophanate-methyl) to control gray mold, strains with the F200Y or E198K mutation that are resistant to both benzimidazole and diethofencarb have been isolated from fields $(15,27)$. Strains with the F200Y mutation are moderately resistant to benzimidazoles, while the E198K mutants like the E198A mutants are highly resistant to benzimidazoles (27). On the other hand, dicarboximides exert their antifungal toxicity by interfering with a histidine-kinase signal transduction pathway $(2,9,10,21-23)$. Mutations in the osmosensor-like histidine kinase $\mathrm{BcOS1}$ gene result in a dicarboximide-resistant phenotype in $B$. cinerea, and strains possessing the I365S mutation in BcOS1p are dominant $(5,15,19,25,26)$. Recently, we scored the sensitivity to iprodione, a dicarboximide fungicide, in $B$. cinerea field isolates collected from several distantly located fields in Japan and identified two additional types of resistant isolates in Osaka area along with the $\mathrm{I} 365 \mathrm{~S}$ mutant isolates (designated as type I). A group of isolates designated type II carried mutations that yield three amino acid substitutions (V368F, Q369H, plus T447S), and the other group designated type III carried mutations causing two amino acid substitutions (Q369P plus N373S) in the BcOS1 gene (25). These three types of dicarboximide-resistant isolates possessed similar levels of resistance and pathogenicity.

A rapid and convenient method to detect fungicide resistance is vital for fungicide resistant management. Advances in molecular biology have provided new opportunities for rapidly detecting 
fungicide-resistant genotypes once the mechanisms of resistance have been elucidated at a molecular level. Several molecular techniques such as allele-specific (AS)-polymerase chain reaction (PCR), PCR-restriction fragment length polymorphism (RFLP), and primer-introduced restriction enzyme analysis (PIRA)-PCR have been reported $(13,17,25)$. These PCR-based methods are more convenient than the traditional antifungal susceptibility test, if only one mutation confers the fungicide resistance. However, at least three types of mutations in the BenA and BcOS1 genes confer resistance to benzimidazoles and dicarboximides, respectively, in $B$. cinerea. In this study, we present single-tube assay protocols for detecting mutations that cause resistance to multiple benzimidazoles as well as dicarboximides. Two adjacent oligonucleotide probes whose fluorescent labels communicate through fluorescence resonance energy transfer are used to recognize a change or changes in the nucleotide sequence of the template DNA by analyzing melting curve profiles on a real-time PCR instrument. The objective of this study was to use this method and (i) analyze the distribution of mutations that confer $B$. cinerea resistance to carbendazim or iprodione in Japan, and (ii) isolate a novel type of benzimidazole-resistant strain that is widely distributed and dominant in Japanese fields.

\section{MATERIALS AND METHODS}

Strains and culture condition. A total of 210 field isolates of $B$. cinerea were used in this study: 105 isolates were previously collected from 26 fields in Japan during 2000 as described previously (25) and the other 105 isolates were newly collected from seven fields in Osaka Prefecture in 2002 (19 isolates), 2003 (21 isolates), 2004 (41 isolates), and 2005 (24 isolates). The five $B$. cinerea strains listed in Table 1 were selected to design the hybridization probe-based PCR protocols for BenA and BcOS1 genotyping. The strain Bc-05-k11 was isolated in Kanagawa Prefecture in 2005. These strains were isolated either from eggplant, tomato, or cucumber plants. B. cinerea strains were maintained on potato dextrose agar (PDA) after single colony isolation.

DNA extraction and sequencing. To extract genomic DNA from the $B$. cinerea isolates, mycelial disks were cultured on cellophane sheets placed on PDA medium at $18^{\circ} \mathrm{C}$ for 3 days.
Mycelia were collected and homogenized with a bead beater (FastPrep Instruction, Qbiogene, Carlsbad, CA) for $30 \mathrm{~s}$ in a lysis buffer $(10 \mathrm{mM}$ Tris- $\mathrm{HCl}$ [pH 8.0], $0.1 \mathrm{M}$ EDTA, and $0.5 \%$ [wt/vol] sodium dodecyl sulfate) and $0.4 \mathrm{~g}$ of glass beads $(1.0 \mathrm{~mm}$ diameter). The resultant sample was incubated at $65^{\circ} \mathrm{C}$ for $30 \mathrm{~min}$. After phenol extraction and isopropanol precipitation, the genomic DNA thus obtained was used as a template for PCR or real-time PCR. DNA sequence analysis was conducted using an ABI Model PRISM310 Auto Sequence System (Applied Biosystems, Foster, CA) and fluorescent dye terminator dideoxynucleotides according to the PCR cycle sequencing protocol provided by the manufacturer. The primer sets for DNA sequencing and PCR amplification were TUB-F1 (5'-GCTTTTGATCTCCAAGATCCG-3'; nucleotide positions, 1757 to 1777) and TUB-R1 (5'-CTGGTCAAAGGAGCAAATCC-3'; nucleotide positions, 2134 to 2115) for BenA (accession no. Z69263) and BcOS1-F1 (5'-TAGCTCATGGAGATCTCACACA-3'; nucleotide positions, 2010 to 2031) and BcOS1-R1 (5'-GACTTCTCGCGCAAATTGTTG-3': nucleotide positions, 2401 to 2381) for BcOS1 (accession no. AF435964).

Real-time PCR using fluorescent hybridization probes. Genotyping of benzimidazole- and dicarboximide-resistant mutations was performed by a LightCycler (LC) instrument (Roche Diagnostics, Basel, Switzerland) with specific sets of primers and hybridization probes (Table 2). These probes consist of two different oligonucleotides - one labeled at the $5^{\prime}$ end with the acceptor fluorophore LC-Red 640 and one labeled at the $3^{\prime}$ end with the donor fluorophore fluorescein. When these probes hybridize to two adjacent internal sequences of the PCR-amplified products and the light source of the LC excites fluorescein, a fluorescent resonance energy transfer occurs between fluorescein and LCRed 640. This results in the emission of fluorescence, which is measured by the instrument. Mutations can be determined by melting curve analyses. Real-time PCR with the hybridization probe was performed in glass capillaries in a total volume of $20 \mu \mathrm{l}$ in the LC system (Roche Diagnostics). Each reaction mixture contained an appropriate amount of genomic DNA, $1 \times$ LC DNA Master Hybridization Probes mix (Roche Diagnostics), $3 \mathrm{mM}$ $\mathrm{MgCl}_{2}, 0.5 \mu \mathrm{M}$ of each primer, $0.2 \mu \mathrm{M}$ of the probe labeled with fluorescein, and $0.25 \mu \mathrm{M}$ of the probe labeled with LC-Red 640. The PCR amplification comprised an initial denaturation step

TABLE 1. List of representative isolates of Botrytis cinerea used in this study

\begin{tabular}{|c|c|c|c|c|c|}
\hline Isolate & $\begin{array}{l}\text { Sensitivity to } \\
\text { carbendazim }^{\text {a }}\end{array}$ & $\begin{array}{l}\text { Sensitivity to } \\
\text { diethofencarb }^{\mathrm{b}}\end{array}$ & $\begin{array}{l}\text { Amino acid substitution } \\
\text { within } \beta \text {-tubulin }\end{array}$ & $\begin{array}{l}\text { Sensitivity to } \\
\text { iprodione }^{\mathrm{c}}\end{array}$ & $\begin{array}{c}\text { Amino acid substitutions } \\
\text { within BcOS } 1\end{array}$ \\
\hline $\mathrm{Bc}-56$ & $\mathrm{~S}$ & $\mathrm{R}$ & $\ldots$ & $\mathrm{S}$ & $\ldots$ \\
\hline $\mathrm{Bc}-45$ & $\mathrm{R}$ & $\mathrm{S}$ & E198A & $\mathrm{R}$ & $\mathrm{I} 365 \mathrm{~S}$ \\
\hline Bc-05-k11 & MR & $\mathrm{R}$ & F200Y & $\mathrm{S}$ & $\ldots$ \\
\hline Bc-M9-3 & $\mathrm{R}$ & $\mathrm{R}$ & E198K & $\mathrm{R}$ & V368F, Q369H, T447S \\
\hline Bc-o-9 & $\mathrm{R}$ & $\mathrm{S}$ & E198A & $\mathrm{R}$ & Q369P, N373S \\
\hline
\end{tabular}

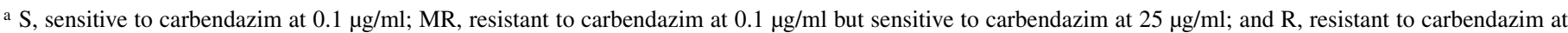
$25 \mu \mathrm{g} / \mathrm{ml}$.

b $\mathrm{S}$, sensitive to diethofencarb at $25 \mu \mathrm{g} / \mathrm{ml}$; and R, resistant to diethofencarb at $25 \mu \mathrm{g} / \mathrm{ml}$.

${ }^{c} \mathrm{~S}$, sensitive to iprodione at $0.4 \mu \mathrm{g} / \mathrm{ml}$; and $\mathrm{R}$, resistant to iprodione at $0.4 \mu \mathrm{g} / \mathrm{ml}$.

TABLE 2. Sequence of the primers and hybridization probes used in the real-time polymerase chain reaction assay of Botrytis cinerea isolates

\begin{tabular}{|c|c|c|c|}
\hline Probe and primer & Sequence $\left(5^{\prime} \rightarrow 3^{\prime}\right)^{\mathrm{a}}$ & Nucleotide position $^{b}$ & Target gene \\
\hline TUB-HPF1 & TGTCGAGCCATATAACGCAA & $1847-1866$ & $\operatorname{BenA}$ \\
\hline TUB-HPR1 & CCAACTTTCGGAGATCTGAG & $2051-2070$ & BenA \\
\hline TUB-U27198-Sn & TGAGAACTCTGACGCGACCTTCTGTAT-FL & $1889-1915$ & $\operatorname{BenA}$ \\
\hline TUB-U27198-An & RED-GATAACGAGGCTCTTTACGATATTTGCATGAGAACC & $1917-1952$ & BenA \\
\hline BcOS1-HPF1 & AGCTCATGGAGATCTCACAC & $2011-2030$ & $\mathrm{BcOS1}$ \\
\hline BcOS1-HPR1 & ATTGGCCATAGCGTTGACGTT & $2219-2239$ & BcOS1 \\
\hline BcOS1-An1 & GTAGGAACTGAAGGTATTCTTGGGGGTCAAG-FL & $2144-2174$ & $\mathrm{BcOS1}$ \\
\hline BcOS1-Sn1 & RED-AGAAATCGAAGGCGTCCAGG & $2176-2195$ & $\mathrm{BcOS1}$ \\
\hline
\end{tabular}

a FL, fluorescein labeled; RED, LC Red 640 labeled.

b Accession number of the BenA ( $\beta$-tubulin) and the BcOS1 (histidine kinase) gene were Z69263 and AF435964, respectively. 
$\left(95^{\circ} \mathrm{C}\right.$ for $\left.30 \mathrm{~s}\right)$ followed by 50 cycles of amplification $\left(95^{\circ} \mathrm{C}\right.$ for $10 \mathrm{~s}, 47^{\circ} \mathrm{C}$ for $10 \mathrm{~s}$, and $72^{\circ} \mathrm{C}$ for $5 \mathrm{~s}$ ) and a melting curve step (denaturation at $95^{\circ} \mathrm{C}$ for $2.5 \mathrm{~min}$; annealing at $40^{\circ} \mathrm{C}$ for $1 \mathrm{~min}$; and melting, increasing temperature to $95^{\circ} \mathrm{C}$ with a slope of 0.1 $\left.\left[{ }^{\circ} \mathrm{C} / \mathrm{s}\right]\right)$.

Assays for fungicide sensitivity and pathogenicity. To determine the sensitivity to fungicides, mycelial disks ( $5 \mathrm{~mm}$ diameter) precultured on PDA medium were placed on PDA and then amended with carbendazim, diethofencarb, or iprodione and cultured at $18^{\circ} \mathrm{C}$ for 3 days. The mean colony diameter minus the diameter of the inoculation disk was then measured and expressed as a percentage of the mean colony diameter of the untreated control. To examine the pathogenicity of $B$. cinerea isolates, mycelial disks (5 $\mathrm{mm}$ diameter) were placed on detached second leaves of cucumber (cv. Sagamihanjiro) as described previously (25) and incubated at $18^{\circ} \mathrm{C}$ for 3 days in a humid, dark chamber. The spreading lesions of $B$. cinerea infection were then photographed.

\section{RESULTS}

Hybridization probe assay to detect three types of benzimidazole-resistant $\boldsymbol{B e n A}$ mutations. It is known that three types of amino acid changes in $\beta$-tubulin $-{ }^{198}$ Glu to Ala (E198A) or Lys (E198K), and ${ }^{200}$ Phe to Tyr (F200Y) - confer benzimidazole resistance in field isolates of $B$. cinerea (27). We screened our stock collection and selected three resistant strains (i.e., Bc45, Bc-M9-3, and Bc-05-k11) possessing the mutations E198A, E198K, and F200Y, respectively, along with a sensitive strain (i.e., Bc-56) without any relevant mutations in the BenA or BcOS1 genes. Their sensitivities to carbendazim, a benzimidazole, and diethofencarb, an $\mathrm{N}$-phenylcarbamate, were associated with the mutation in $\beta$-tubulin (Table 1) and were essentially identical to those described by Yarden and Katan (27). The strains Bc-45 (E198A) and Bc-M9-3 (E198K) grew on the medium containing carbendazim at $25 \mu \mathrm{g} / \mathrm{ml}$, while the strain Bc-56 was sensitive to carbendazim at $0.1 \mu \mathrm{g} / \mathrm{ml}$. In contrast, the strain Bc-05-k11 (F200Y) was moderately resistant to carbendazim because it grew on the medium containing carbendazim at $0.1 \mu \mathrm{g} / \mathrm{ml}$ but not carbendazim at $25 \mu \mathrm{g} / \mathrm{ml}$ (Table 1). The strain Bc-45 was sensitive to diethofencarb at $0.1 \mu \mathrm{g} / \mathrm{ml}$, but the other strains including the sensitive Bc-56 strain were highly resistant to diethofencarb.

We indicated that amino acid substitutions associated with the benzimidazole-resistant phenotype occur at amino acid positions 198 and 200 in $\beta$-tubulin, and their mutation sites are not more than 9 bases in length. The presence of mutations within a narrow region implied that a single hybridization probe can cover these mutation sites and distinguish each mutation by analyzing the melting curve profiles after PCR amplification of the target sequence followed by hybridization. We designed PCR primers and hybridization probes to detect the BenA mutations in real-time PCR as shown in Table 2 and Figure 1A. The primers TUB-HPF1 and TUB-HPR1 were designed to specifically amplify a 224-bp region of the BenA gene that includes the 198 and 200th codons. A sensor probe (i.e., TUB-U27198-Sn) labeled at the $3^{\prime}$ end with fluorescein can be hybridized to a region containing the mutation sites, and an anchor probe (i.e., TUB-U27198-An) labeled at the 5 ' end with LC-Red 640 can be hybridized $1 \mathrm{bp}$ from the sensor probe. Therefore, two fluorophores localize close enough to yield a fluorescent resonance energy transfer. When a thermal analysis was performed using each type of the BenA gene, the melting peak temperature depended on the homology between the sensor probe and the amplified PCR product. As shown in Figure 1B, this sensor probe produced discernible melting peaks at 68.7, $62.0,59.4$, and $57.2^{\circ} \mathrm{C}$ for the BenA genes bearing the E198A mutation (GAG $\rightarrow \mathrm{GCG}$ ), the wild-type sequence, the E198K mutation $(\mathrm{GAG} \rightarrow \underline{\mathrm{A} A G})$, and the F200Y mutation (TTC $\rightarrow$ T $\underline{\mathrm{AC}}$ ), respectively. The PCR fragments containing the E198A mutation (a perfect match with the sensor probe) formed the most stable hybrid with the sensor probe and showed the highest melting peak temperature. Utilizing another sensor probe with a wild-type sequence failed to produce discernible melting curve profiles (data not shown). These data indicate that the hybridization probe assay using real-time PCR is a simple and rapid method to detect multiple benzimidazole-resistant mutations in a single PCR reaction if the hybridization probes are properly designed.

Hybridization probe assay to detect three types of dicarboximide-resistant $\mathrm{BcOS1}$ mutations. Mutations in the $\mathrm{BcOS1}$ gene that encode a histidine kinase confer dicarboximide resistance to field isolates $(5,15,19,26)$. We previously reported that the strains with the ${ }^{365}$ Ile to Ser (I365S) mutation (type I) were dominant in Japan; however, two other types of resistant strains, represented by $\mathrm{Bc}-\mathrm{M} 9-3$ and $\mathrm{Bc}-\mathrm{O}-9$, were isolated mainly from the Osaka area (25). These two strains have three amino acid substitutions $\left[{ }^{368}\right.$ Val to Phe (V368F), ${ }^{369} \mathrm{Gln}$ to His $(\mathrm{Q} 369 \mathrm{H})$, plus ${ }^{447} \mathrm{Thr}$ to Ser (T447S) (type II)] and two amino acid substitutions $\left[{ }^{369}\right.$ Gln to Pro (Q369P) plus ${ }^{373}$ Asn to Ser (N373S) (type III)], respectively, in BcOS1p (Table 1). Most of known dicarboximideresistant field strains have mutation(s) in the region between the 365 th and the 369th codon of the BcOS1 gene, suggesting that mutations in this region confer dicarboximide resistance in $B$. cinerea. Therefore, we designed a sensor probe, i.e., BcOS1Sn1, labeled at the $5^{\prime}$ end with LC-Red 640 that surveys the region containing three types of mutations-I365S (ATC $\rightarrow$ A $\underline{G C}$ ), V368F $(\mathrm{GTC} \rightarrow$ TTC) plus Q369H $(\mathrm{CAG} \rightarrow \mathrm{CAC})$, and Q369P $(\mathrm{CAG} \rightarrow \mathrm{C} \underline{\mathrm{CG}}$ ) (Fig. 1C). After PCR amplification of the target sequence of the BcOS1 gene with the primers BcOS1-HPF1 and BcOS1-HPR1, thermal analyses were performed using the hybridization probes $\mathrm{BcOS} 1-\mathrm{Sn} 1$ and BcOS1-An1. Melting curve peaks were detected at $65.7^{\circ} \mathrm{C}$ (Bc-56, wild-type), $62.5^{\circ} \mathrm{C}$ (Bc-o9, Q369P), 59.2 ${ }^{\circ} \mathrm{C}$ (Bc-45, I365S), and $54.5^{\circ} \mathrm{C}$ (Bc-M9-3, V368F, and Q369H) (Fig. 1D). BcOS1-Sn1, whose sequence was identical to that of the wild-type $B C O S 1$ gene, formed the most stable hybrid with the PCR products amplified from the wild-type BcOS1 gene and formed the most unstable hybrid with the PCR products amplified from the $\mathrm{BcOS} 1$ gene in Bc-M9-3. These data indicate that this hybridization probe assay using real-time PCR is useful to detect not only the mutations responsible for benzimidazole resistance but also those responsible for dicarboximide resistance.

Genotyping of $B$. cinerea field isolates revealed a wide distribution of a novel type of benzimidazole-resistant strain in Japan. A total of $105 \mathrm{~B}$. cinerea field isolates were collected from 26 fields in nine distantly located regions in Japan (25). We attempted to classify the BcOS1 and BenA genotypes of these 105 isolates using newly designed hybridization probe assay protocols. In BcOS1 genotyping, the melting peak temperature, obtained from the genomic DNA of each isolate used as a template, matched that from one of four standard $B c O S 1$ genes (sensitive and three types of mutants) without exception, and the mutations in the BcOS1 genes were identical to those determined by DNA sequencing, PCR-RFLP, and/or AS-PCR methods, as previously described (25). However, the presence of an unassigned melting peak at $63.4^{\circ} \mathrm{C}$ in the BenA genotyping assay (Fig. 1B) suggested the presence of another type of mutation in the BenA gene that may confer resistance to benzimidazole. DNA sequencing analysis revealed that the BenA genes with the unassigned peak under the melting curve assay had a point mutation $(\mathrm{GAG} \rightarrow \mathrm{GTG})$ at codon 198 in which glutamic acid was substituted by valine (E198V). Six isolates with the BenA E198V mutation were selected (Bc-c13-3 and Bc-c13-7 from Chiba, Bc-o-36 and Bc-o18 from Osaka, Bc-k-18 from Kochi, and Bc-m-1 from Miyazaki) and the sensitivity to carbendazim, diethofencarb, and iprodione (Table 3) was examined. All isolates with the BenA E198V mutation grew well on a medium amended with carbendazim at $25 \mu \mathrm{g} / \mathrm{ml}$, similar to the strains with the E198A or E198K muta- 
tion, suggesting that glutamic acid at codon 198 is essential for sensitivity to benzimidazoles. Negative cross-resistance to diethofencarb was observed in the isolates with the BenA E198V mutation; however, they were less sensitive to diethofencarb compared with the strains with the BenA E198A mutation (Table 3). The values of $\mathrm{EC}_{50}$ (concentration required to inhibit radial growth by $50 \%$ ) for diethofencarb ranged from 0.7 to $0.8 \mu \mathrm{g} / \mathrm{ml}$ in the six examined isolates with BenA E198V while that for the Bc-45 strain with BenA E198A was $0.1 \mu \mathrm{g} / \mathrm{ml}$. The Bc-o-18 isolate with the BenA E198V mutation infected cucumber leaves, and its lesions expanded in high relative humidity (>95\% RH) (Fig. 2). The size of the lesions in the Bc-o-18 isolate was very similar to those of the benzimidazole-sensitive and -resistant strains, suggesting that strains with the BenA E198V mutation have a pathogenicity similar to that of other strains. The E198V mutation in the BenA gene had no apparent effect on the sensitivity to iprodione. Two isolates (Bc-c13-3 and $\mathrm{Bc}-\mathrm{O}-36)$ possessing the wildtype $\mathrm{BcOS} 1$ gene and four isolates (Bc-c13-7, Bc-o-18, Bc-k-18, and $\mathrm{Bc}-\mathrm{m}-1)$ with the I365S mutation showed sensitivities similar to those of the Bc-56 (sensitive to iprodione) and Bc-45 (resistant to iprodione) strains, respectively (Table 3 ).

A devised genotyping assay protocol was used to survey the occurrence of benzimidazole-resistant BenA mutations in B. cinerea in Japan (Table 4). Among the 105 field isolates, 70 isolates were
A

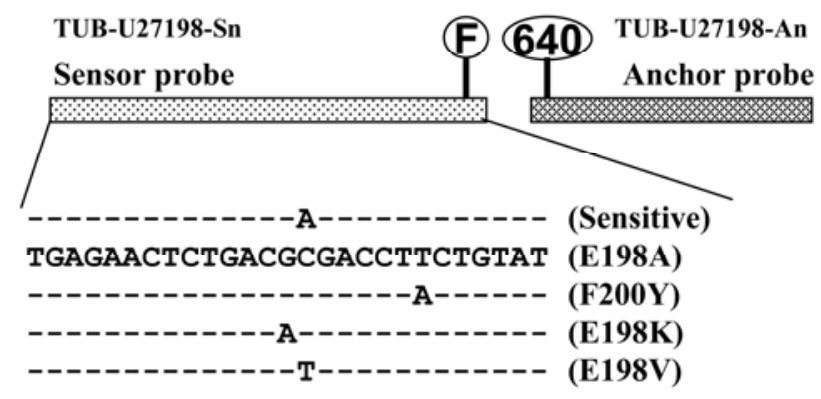

C

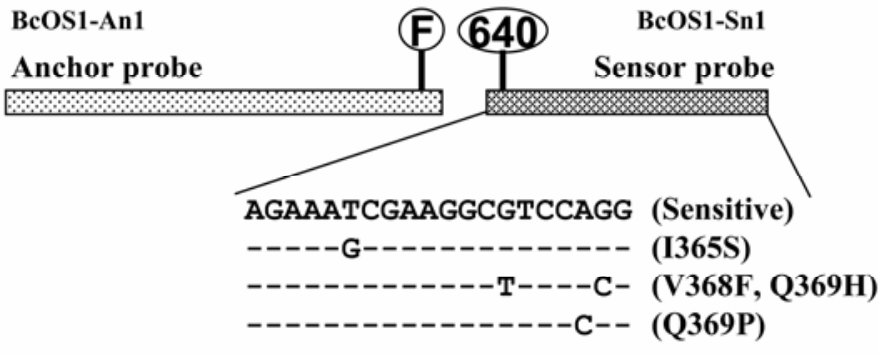

B

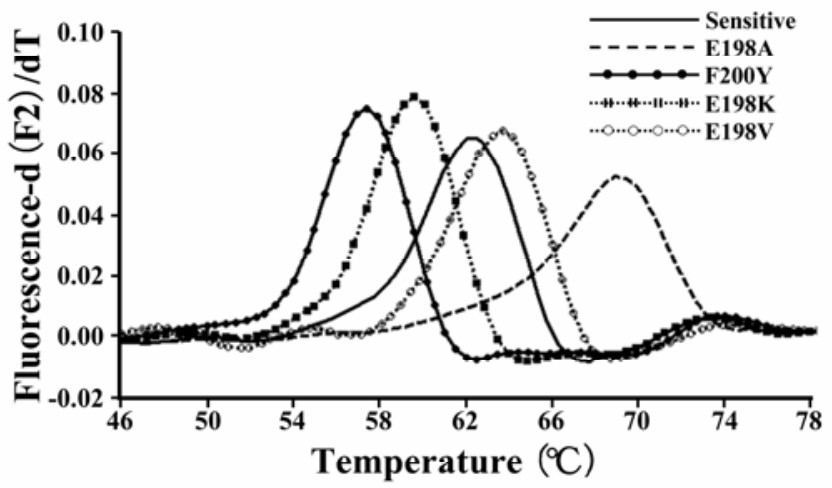

D

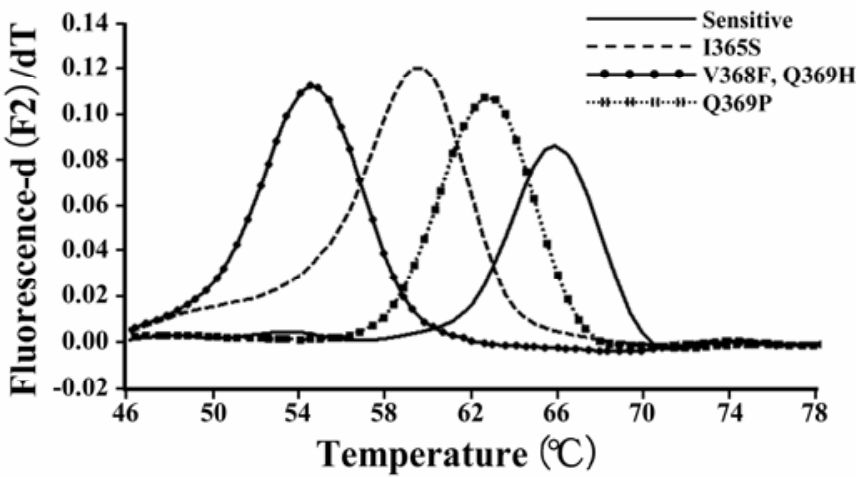

Fig. 1. Real-time polymerase chain reaction using fluorescent hybridization probes and melting curve analysis. A, DNA sequence of the sensor probe and mutations conferring benzimidazole resistance in the $\beta$-tubulin BenA gene. Hyphens (-) indicate nucleotides identical to those in the sensor probe. The sensor probe TUB-U27198-Sn is a perfect match to the BenA E198A gene. Therefore, the wild-type BenA gene and the F200Y, E198K, and E198V mutants have a single mismatch, i.e., $\mathrm{C} \rightarrow \mathrm{A}, \mathrm{T} \rightarrow \mathrm{A}, \mathrm{G} \rightarrow \mathrm{A}$, and $\mathrm{C} \rightarrow \mathrm{T}$, respectively. B, Derivative melting curves $[-(\mathrm{dF} / \mathrm{dT})$ versus $\mathrm{T}]$ of the wild-type BenA gene and the four types of benzimidazole-resistant mutations for the BenA genes. The fluorescence signal $(\mathrm{F})$ was continuously monitored during the temperature ramp and then plotted against temperature ( $\mathrm{T}$ ) to obtain the melting curves for the samples (F versus $\mathrm{T}$ ). The melting curves were subsequently converted to derivative melting curves [-(dF/dT) versus T]. The melting peaks of the wild-type BenA gene and those for the E198A, E198V, E198K, and F200Y mutants were at 62.0, 68.7, 63.4, 59.4, and $57.2^{\circ} \mathrm{C}$, respectively. $\mathbf{C}$, DNA sequence of the sensor probe and the mutations conferring dicarboximide resistance in the $B c O S 1$ gene. Hyphens (-) indicate nucleotides identical to those of the sensor probe. The sensor probe BcOS1-Sn1 is a perfect match to the wild-type (sensitive) $B c O S 1$ gene. The BcOS1 genes with the I365S and Q369P mutations have single mismatches, i.e., $\mathrm{T} \rightarrow \mathrm{G}$ and $\mathrm{A} \rightarrow \mathrm{C}$, respectively. The BcOS1 gene with the V368F plus Q369H mutations has two mismatches, i.e., $\mathrm{G} \rightarrow \mathrm{T}$ and $\mathrm{G} \rightarrow \mathrm{C}$. D, Derivative melting curve of the wild-type $\mathrm{BcOS1}$ gene and three types of dicarboximide-resistant mutations in the $\mathrm{BcOS1}$ gene. The melting peaks of the wild-type $B c O S 1$ gene and the Q369P, I365S, and V368F plus Q369H mutations were at $65.7,62.5,59.2$, and $54.5^{\circ} \mathrm{C}$, respectively.

TABLE 3. Sensitivity to fungicides in benzimidazole-resistant isolates of Botrytis cinerea with E198V in BenA gene

\begin{tabular}{lcccc}
\hline & & & \multicolumn{2}{c}{ Sensitivity to fungicides $^{\mathrm{a}}$} \\
\cline { 3 - 5 } Isolate & Amino acid substitution & Carbendazim & Diethofencarb & Iprodione \\
\hline Bc-56 & Sensitive & 0.1 & $>25$ & 0.1 \\
Bc-45 & E198A & $>25$ & $>25$ & 2.6 \\
Bc-05-k11 & F200Y & 6.6 & $>25$ & 0.2 \\
Bc-M9-3 & E198K & $>25$ & 0.7 & 2.2 \\
Bc-c13-3, Bc-o-36 & E198V & $>25$ & $0.7-0.8$ & 0.2 \\
Bc-c13-7, Bc-o-18, Bc-k-18, Bc-m-1 & E198V & $>25$ & $2.0-2.5$ & \\
\hline
\end{tabular}

${ }^{a}$ Concentration that inhibits radial growth by $50 \%\left(\mathrm{EC}_{50}\right)$. 
resistant to carbendazim, 32 isolates possessed the E198A mutation, 37 isolates possessed the E198V mutation, and one isolate possessed the E198K mutation. This result indicated that E198V and E198A were two major benzimidazole-resistant mutations. The strains carrying these two mutations were widely distributed in Japan, whereas strains with the E198V mutation were not found in Hokkaido Prefecture, a northern island of Japan (data not shown). On the other hand, we found only one isolate with the E198K mutation and no isolate with the F200Y mutation in 105 isolates. We have reported that these $105 \mathrm{~B}$. cinerea field isolates can be grouped into four categories according to their $\mathrm{BcOS1}$ genotype by procedures based on PCR-RFLP and AS-PCR (25). The combination of BenA and BcOS1 genotyping assays classified $B$. cinerea into 20 groups. Twenty-seven isolates with the BenA E198V mutation had the BcOS1 I365S mutation, and 10 other isolates with the E198V mutation had no mutation in the $B c O S 1$ gene and were sensitive to dicarboximide. No BcOS1 Q369P or V368F plus Q369H mutants were found in the BenA E198V mutants.

Regional survey of genotype changes in $\boldsymbol{B}$. cinerea. We previously reported that dicarboximide-resistant strains with the $B c O S 1$ I365S mutation were distributed throughout Japan, but other types of resistant strains with the BcOS1 Q369P and BcOS1 V368F plus Q369H mutations were almost specifically found in fields in Osaka during 2000 (Table 4) (25). To determine the stability of these unique dicarboximide-resistant strains in the Osaka

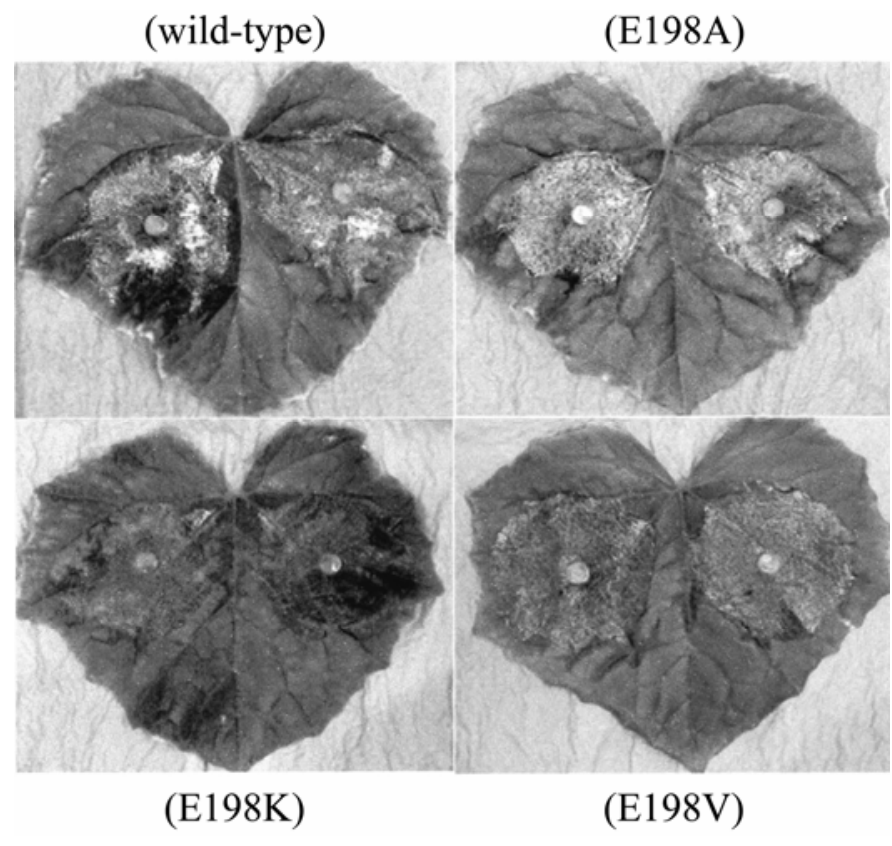

Fig. 2. Pathogenicity of Botrytis cinerea with benzimidazole-resistant mutant alleles on detached cucumber leaves. The sensitive (Bc-56) and benzimidazole-resistant Bc-45 (E198A), Bc-M9-3 (E198K), and Bc-o-18 (E198V) isolates were incubated on potato dextrose agar plates at $18^{\circ} \mathrm{C}$ for 7 days. Their mycelial disks ( $5 \mathrm{~mm}$ diameter) were placed on the detached first leaves of cucumber and incubated at $18^{\circ} \mathrm{C}$ for 3 days in a humid, dark chamber. area, we further obtained a total of $105 \mathrm{~B}$. cinerea strains (40 isolates between 2002 and 2003 and 65 isolates between 2004 and 2005). Mutations within the BcOS1 and BenA genes were determined using hybridization probe assay procedures described previously (Table 5). The proportion of dicarboximide-sensitive isolates was $17 \%$ in $2000,43 \%$ from 2002 to 2003 , and $45 \%$ from 2004 to 2005 . The increasing frequency of dicarboximide-sensitive isolates probably come from the reducing usage of dicarboximide fungicides by introduction of new fungicides such as phenylpyrroles, anilinopyrimidines, and hydroxyanilides. An increased proportion of dicarboximide-sensitive isolates in the Osaka area mainly resulted from an increase in the number of isolates that were sensitive to both carbendazim and iprodione. The strain bearing the BcOS1 I365S mutation was a major dicarboximideresistant population in every period. The $B c O S 1$ I365S mutation was only found in benzimidazole-resistant isolates, and most of these isolates also carried the BenA E198V mutation. The proportion of the $B c O S 1 \mathrm{~V} 368 \mathrm{~F}$ plus Q369H isolates in the total isolates decreased 34\% (10 of 29) in 2000, 13\% (5 of 40) from 2002 to 2003, and 6\% (4 of 65) from 2004 to 2005. Most of the BcOS1 V368F plus Q369H isolates (16 of 19) were sensitive to carbendazim. Meanwhile, the ratio of the number of benzimidazolesensitive to the total number of isolates was stable at 32 to $35 \%$ from 2000 to 2005. Only two isolates possessing the BcOS1 Q369P mutation along with the E198A mutation in the BenA gene had been isolated in 2000 and were not isolated thereafter. The strain with the BcOS1 Q369P and the BenA E198V mutations was not isolated in 2000 but became dominant as its proportion increased to 31 and $28 \%$ in the benzimidazole-resistant isolates between 2002 and 2003 and 2004 and 2005, respectively. Moreover, all isolates with the BcOS1 Q369P mutation carried the BenA E198V mutation. These results indicated that the dicarboximide-resistant strains with the $B c O S 1 \mathrm{~V} 368 \mathrm{~F}$ plus Q369H and the $B c O S 1$ Q369P mutations appeared in different strains with the $B e n A$ genotype in the fields in Osaka. The benzimidazoleresistant strains with the E198K or F200Y mutation in the BenA gene were also seldom isolated, suggesting that these types of benzimidazole-resistant strains exist at a very low rate.

\section{DISCUSSION}

We have developed hybridization probe assay procedures for the rapid identification of base substitutions in the $\beta$-tubulin BenA gene and the histidine kinase $B c O S 1$ gene that are associated with resistance to benzimidazole and dicarboximide fungicides in $B$. cinerea, respectively. Our method is based on the hybridization property of DNA and a fluorescent resonance energy transfer between two fluorophores. Fluorescence emission occurs if two hybridization probes (sensor and anchor) are closely located. Anchor probes are designed to hybridize to the template DNA at a higher melting temperature than sensor probes. In thermal analysis, a hybrid between the sensor probe and the template with base substitution(s) results in an unstable hybrid that becomes dehybridized at a lower temperature. The peak of the melting curve that is drawn differentiating fluorescence and time versus temperature depends on the homology of the probe and the template. The position of a base substitution may influence the stability of

TABLE 4. Genotyping of benzimidazole- and dicarboximide-resistant isolates of Botrytis cinerea in Japan

\begin{tabular}{|c|c|c|c|c|c|c|}
\hline \multirow[b]{2}{*}{ BcOS1 genotype } & \multicolumn{6}{|c|}{ Number of isolates with $B e n A$ mutation $^{\mathrm{a}}$} \\
\hline & Wild type & E198A & F200Y & E198K & E198V & Total \\
\hline Wild type & $24(2)$ & $15(2)$ & 0 & 0 & $10(1)$ & $49(5)$ \\
\hline $\mathrm{I} 365 \mathrm{~S}$ & 3 & $13(2)$ & 0 & 0 & $27(10)$ & $43(12)$ \\
\hline V368F, Q369H & $8(8)$ & $2(2)$ & 0 & 1 & 0 & $11(10)$ \\
\hline Q369P & 0 & $2(2)$ & 0 & 0 & 0 & $2(2)$ \\
\hline Total & $35(10)$ & $32(8)$ & 0 & 1 & $37(11)$ & $105(29)$ \\
\hline
\end{tabular}

a Numbers of Osaka isolates are indicated in parenthesis. 
the hybrid, and even a base change at the same position can alter the stability. In fact, the BenA E198A and E198V mutations each possess a base change at the same position, and the hybridization of the TUB-U27198-Sn sensor probe with the E198A sequence distinguishes between the wild-type BenA, the BenA E198V, and the BenA E198A genes (Fig. 1A and B).

Recently, several PCR-based procedures for the detection of fungicide-resistant mutations have been successfully used to identify mutations conferring resistance to fungicides, including benzimidazoles and dicarboximides, in many plant pathogens (12, $18,19,25)$. We also previously reported two PCR techniques for the detection of the dicarboximide-resistant $B c O S 1$ mutation in B. cinerea; the I365S mutation was detected by PCR-RFLP and the V368F plus Q369H mutations could be detected by AS-PCR. In addition, the Q369P plus N373S mutations were identified by direct DNA sequencing (25). As described herein, three types of dicarboximide-resistant isolates with mutation(s) in the BcOS1 gene and four types of benzimidazole-resistant isolates with mutation(s) in the BenA genes were found in Japanese fields. As we need to detect each type of mutation with less effort, preferably in a single reaction, the hybridization probe assays developed in this study for BcOS1 and BenA genotyping have clear advantages over the PCR-RFLP and/or AS-PCR procedures. In a PCR-based hybridization probe methodology, the amplification of template DNA and the detection of mutations are carried out in a single tube with no additional manipulations such as restriction digestion and gel electrophoresis after the PCR set-up. Furthermore, this method can identify the presence of unidentified mutation. In addition, genotyping based on the melting curve analysis could clearly detect the individual mutations from a mixture of DNAs and estimate the ratios of strains, if the difference of peak temperature is more than $5^{\circ} \mathrm{C}$. For example, a mixture of DNA from dicarboximide-sensitive (wild-type) and -resistant (I365S) isolates would display two melting peaks and the intensities depend on the population of their source. Their melting temperatures are in agreement with those from the individual $B c O S 1$ genotype assays (S. Banno and M. Fujimura, unpublished data).

A novel benzimidazole-resistant mutation, E198V, in the BenA gene was identified in $B$. cinerea. This mutation was initially discovered at an unassigned melting curve peak during BenA genotyping (Table 3). Changes at codons 198 and 200 in the $\beta$ tubulin gene are responsible for benzimidazole resistance in most field isolates of plant-pathogenic fungi $(14,15,19,20)$. The E198A and E198K mutations were found in the benzimidazole-resistant isolates of many plant pathogens; however, the E198V mutation was reported only in benzimidazole-resistant Penicillium expan- sum (14). Mutational analysis of the $\beta$-tubulin $\mathrm{Bml}$ gene in Neurospora crassa indicated that a glutamic acid residue at position 198 of $\beta$-tubulin has the size and charge required for binding to the benzimidazoles, and a smaller amino acid and loss of polarity facilitates diethofencarb binding to $\beta$-tubulin (8). B. cinerea strains with the E198V mutation as well as the E198A and E198K mutations were highly resistant to the benzimidazole carbendazim (Table 3). Interestingly, B. cinerea isolates with the E198V mutation were distributed widely in Japan (Table 5). It is known that the application of benzimidazoles to control gray mold leads to the appearance of resistant isolates with the E198A mutation that show negative cross-resistance to diethofencarb. The application of a mixture of carbendazim (or thiophanate-methyl) and diethofencarb may yield strains with the E198K or F200Y mutations in the BenA gene (15). B. cinerea isolates with the E198K or F200Y mutations were barely found in our survey, even though the pathogenicity of isolates with these mutations on cucumber leaves were very similar to that of the other BenA mutants or the wildtype strain (Fig. 2). The fungicide programs for gray mold control have been shifted from the use of mixtures of diethofencarb and carbendazim or thiophanate-methyl to new classes of fungicides such as phenylpyrroles, anilinopyrimidines, and hydroxyanilides. The isolates bearing the E198K or F200Y mutations might be less fit than the wild-type strains or resistant isolates with the E198A or E198V mutation in the absence of fungicide selection.

We previously reported that dicarboximide-resistant strains with the BcOS1 I365S mutation are widely spread throughout Japan, and the other two types of dicarboximide-resistant mutations were detected among isolates in the Osaka area collected in 2000; one isolate has three amino acid substitutions (V368F, Q369H, plus T447S) and the other isolate has two amino acid substitutions (Q369P plus N373S) within the histidine kinase BcOS1p (Table 5). The BcOS1 genotyping of field isolates collected from 2002 to 2005 revealed that these two types of dicarboximide-resistant strains were established in the Osaka area. Ma et al. (19) reported that some dicarboximide-resistant strains isolated in California carried the Q369P mutation in the BcOS1 gene; this may suggest the global distribution of this mutation. Other types of dicarboximide-resistant mutations in the $B c O S 1$ gene that cause a single amino acid substitution (I365N or I365R) instead of I365S were found in New Zealand, Europe, and the United States $(5,15,19)$. Additionally, a strain with two amino acid substitutions (i.e., I365S and Q369P) was found in Pukekohe, New Zealand (5). We synthesized three single-stranded oligonucleotides whose nucleotide sequences matched either those of the BcOS1 I365N, I365R, or I365S plus Q369P mutations and

TABLE 5. Genotyping of benzimidazole- and dicarboximide-resistant isolates of Botrytis cinerea from Osaka fields in 2000, 2002-2003, and 2004-2005

\begin{tabular}{|c|c|c|c|c|c|c|}
\hline \multirow[b]{2}{*}{ Year(s)/BcOS1 genotype } & \multicolumn{6}{|c|}{ Number of isolates with $B e n A$ mutation } \\
\hline & Wild type & E198A & F200Y & E198K & E198V & Total \\
\hline \multicolumn{7}{|l|}{2000} \\
\hline Wild type & 2 & 2 & 0 & 0 & 1 & 5 \\
\hline $\mathrm{I} 365 \mathrm{~S}$ & 0 & 2 & 0 & 0 & 10 & 12 \\
\hline V368F, Q369H & 8 & 2 & 0 & 0 & 0 & 10 \\
\hline Q369P & 0 & 2 & 0 & 0 & 0 & 2 \\
\hline Total & 10 & 8 & 0 & 0 & 11 & 29 \\
\hline \multicolumn{7}{|l|}{$2002-2003$} \\
\hline Wild type & 9 & 5 & 0 & 0 & 3 & 17 \\
\hline $\mathrm{I} 365 \mathrm{~S}$ & 0 & 5 & 0 & 0 & 5 & 10 \\
\hline V368F, Q369H & 5 & 0 & 0 & 0 & 0 & 5 \\
\hline Q369P & 0 & 0 & 0 & 0 & 8 & 8 \\
\hline Total & 14 & 10 & 0 & 0 & 16 & 40 \\
\hline \multicolumn{7}{|l|}{ 2004-2005 } \\
\hline Wild type & 18 & 5 & 0 & 0 & 6 & 29 \\
\hline $\mathrm{I} 365 \mathrm{~S}$ & 0 & 0 & 1 & 0 & 19 & 20 \\
\hline V368F, Q369H & 3 & 1 & 0 & 0 & 0 & 4 \\
\hline Q369P & 0 & 0 & 0 & 0 & 12 & 12 \\
\hline Total & 21 & 6 & 1 & 0 & 37 & 65 \\
\hline
\end{tabular}


were used as a template for thermal analysis to obtain the melting curve profiles of these mutants. The peak temperatures of these dicarboximide-resistant mutants did not match any of those of the four types of Japanese isolates used in this study (data not shown). Therefore, we conclude that all the isolates we collected from the Japanese fields belong to one of four types regarding the BcOS1 gene. Recently, a new dicarboximide-resistant BcOS1 mutation which causes immature termination at amino acid position 1040 of BcOS1p was found in one isolate in the United States (19), yet obviously our protocol is not designed to detect this mutation.

The hybridization assays developed enable the classification of $B$. cinerea into a total of 20 types (Tables 4 and 5). Genotyping analyses of the BenA and $B c O S 1$ genes provide important information for the selection of fungicides for the control of gray mold. First, a mixture of a benzimidazole fungicide, carbendazim or thiophanate-methyl, with diethofencarb controls the wild-type isolates and isolates with the E198A and probably the E198V mutations in the BenA gene but not those with the E198K or F200Y mutation (Table 3). Second, dicarboximide-resistant isolates frequently carry the benzimidazole-resistant mutation, particularly in isolates with the BcOS1 I365S mutation. In other words, only a small portion of the isolates were sensitive to benzimidazoles and resistant to dicarboximides. Similar results were reported for $B$. cinerea collected in South Carolina $(28,29)$. These observations suggested that not only a particular mutation in $B e n A$ and $B c O S 1$ but also their combination may affect the fitness and stability of $B$. cinerea isolates in the field.

The hybridization assay based on PCR amplification and thermal analysis is a reliable detection procedure that does not involve postamplification steps such as restriction enzyme digestion and electrophoresis of the DNA fragment. This method based on the use of a fluorescent hybridization probe in real-time PCR has been successfully applied in the mutational analysis of several drug-resistant clinical pathogens $(4,16,24)$. In conclusion, we emphasize that such hybridization assays could be useful for the detection of mutations resistant to other fungicides in various pathogens with clear advantages. The commonly used PCR-based method specifically detects only one type of mutation; however, the dissociation curve analysis in the hybridization probe assay may have the potential to detect any mutation within the annealing region of the probes. Therefore, this method would be applicable for the detection of mutations resistant to new fungicides such as the mitochondria complex III inhibitor (quinone outside inhibitors) in various pathogens.

\section{ACKNOWLEDGMENTS}

This work was supported in part by the University-Industry Joint Research Project for Private Universities with a matching fund subsidy from the Ministry of Education, Culture, Sports, Science, and Technology of Japan, and in part by the Research Project for Utilizing Advanced Technologies in Agriculture, Forestry, and Fisheries.

\section{LITERATURE CITED}

1. Altertini, C., Gredt, M., and Leroux, P. 1999. Mutations of the $\beta$-tubulin gene associated with different phenotypes of benzimidazole resistance in the cereal eyespot fungi Tapesia yallundae and Tapesia acuformis. Pestic. Biochem. Physiol. 64:17-31.

2. Banno, S., Noguchi, R., Yamashita, K., Fukumori, F., Kimura, M., Yamaguchi, I., and Fujimura, M. 2007. Roles of putative His-to-Asp signaling modules HPT-1 and RRG-2, on viability and sensitivity to osmotic and oxidative stresses in Neurospora crassa. Curr. Genet. 51:197-208.

3. Beever, R. E., Laracy, E. P., and Pak, H. A. 1989. Strains of Botrytis cinerea resistant to dicarboximide and benzimidazole fungicides in New Zealand vineyards. Plant Pathol. 38:427-437.

4. Carattoli, A., Dionisi, A., and Luzzi, I. 2002. Use of a LightCycler gyrA mutation assay for identification of ciprofloxacin-resistant Campylobacter coli. FEMS Microbiol. Lett. 214:87-93.

5. Cui, W., Beever, R. E., Parkes, S. L., and Templeton, M. D. 2004. Evolution of an osmosensing histidine kinase in field strains of Botryotinia fuckeliana (Botrytis cinerea) in response to dicarboximide fungicide usage. Phytopathology 94:1129-1135.

6. Davidse, L. C., and Flach, W. 1978. Interaction of thiabendazole with fungal tubulin. Biochim. Biophys. Acta 543:82-90.

7. Fujimura, M., Kamakura, T., Inoue, H., and Yamaguchi, I. 1994. Aminoacid alterations in the beta-tubulin gene of Neurospora crassa that confer resistance to carbendazim and diethofencarb. Curr. Genet. 25:418-422.

8. Fujimura, M., Kamakura, T., Yamaguchi, I. 1994. Mode of action of diethofencarb to benzimidazole-resistant mutant in Neurospora crassa. J. Pestic. Sci. 17:237-242.

9. Fujimura, M., Ochiai, N., Motoyama, T., and Yamaguchi, I. 2002. Mechanism of resistance to dicarboximides and phenylpyrrols. Pages 200-214 in: Agrochemical Resistance; Extent, Mechanism, and Detection. J. M. Clark and I. Yamaguchi, ed. American Chemical Society, Washington, DC.

10. Fujimura, M., Ochiai, N., Oshima, M., Motoyama, T., Ichiishi, A., Usami, R., Horikoshi, K., and Yamaguchi, I. 2003. Putative homologs of SSK22 MAPKK kinase and PBS2 MAPK kinase of Saccharomyces cerevisiae encoded by os -4 and $o s-5$ genes for osmotic sensitivity and fungicide resistance in Neurospora crassa. Biosci. Biotechnol. Biochem. 67:186191.

11. Fujimura, M., Oeda, K., Inoue, H., and Kato, T. 1992. A single aminoacid substitution in the beta-tubulin gene of Neurospora confers both carbendazim resistance and diethofencarb sensitivity. Curr. Genet. 21:399-404.

12. Ishii, H. 2006. Impact of fungicide resistance in plant pathogens on crop disease control and agricultural environment. JARQ 40:205-211.

13. Kaku, K., Takagaki, M., Shimizu, T., and Nagayama, K. 2003. Diagnosis of dehydratase inhibitors in melanin biosynthesis inhibitor (MBI-D) resistance by primer-introduced restriction enzyme analysis in scytalone dehydratase gene of Magnaporthe grisea. Pest Manag. Sci. 59:843-846.

14. Koenraadt, H., Somerville, S. C., and Jones, A. L. 1992. Characterization of mutations in the beta-tubulin gene of benomyl-resistant field strains of Venturia inaequalis and other plant pathogenic fungi. Phytopathology 82:1348-1354.

15. Leroux, P., Fritz, R., Debieu, D., Albertini, C., Lanen, C., Bach, J., Gredt, M., and Chapeland, F. 2002. Mechanisms of resistance to fungicides in field strains of Botrytis cinerea. Pest Manag. Sci. 58:876-888.

16. Loeffler, J., Hagmeyer, L., Hebart, H., Henke, N., Schumacher, U., and Einsele, H. 2000. Rapid detection of point mutations by fluorescence resonance energy transfer and probe melting curves in Candida species. Clin. Chem. 46:631-635.

17. Luck, J. E., and Gillings, M. R. 1995. Rapid identification of benomyl resistant strains of Botrytis cinerea using the polymerase chain reaction. Mycol. Res. 99:1483-1488.

18. Ma, Z., and Michailides, T. J. 2005. Advances in understanding molecular mechanisms of fungicide resistance and molecular detection of resistant genotypes in phytopathogenic fungi. Crop Prot. 24:853-863.

19. Ma, Z., Yan, L., Luo, Y., and Michailides, T. J. 2007. Sequence variation in the two-component histidine kinase gene of Botrytis cinerea associated with resistance to dicarboximide fungicides. Pestic. Biochem. Physiol. 88:300-306.

20. Maymon, M., Zveibil, A., Pivonia, S., Minz, D., and Freeman, S. 2006. Identification and characterization of benomyl-resistant and -sensitive populations of Colletotrichum gloeosporioides from Statice (Limonium spp.). Phytopathology 96:542-548.

21. Motoyama, T., Ohira, T., Kadokura, K., Ichiishi, A., Fujimura, M., Yamaguchi, I., and Kudo, T. 2005. An Os-1 family histidine kinase from a filamentous fungus confers fungicide-sensitivity to yeast. Curr. Genet. 47:298-306

22. Noguchi, R., Banno, S., Ichikawa, R., Fukumori, F., Ichiishi, A., Kimura, M., Yamaguchi, I., and Fujimura, M. 2007. Identification of OS-2 MAP kinase-dependent genes induced in response to osmotic stress, antifungal agent fludioxonil, and heat shock in Neurospora crassa. Fungal Genet. Biol. 44:208-218.

23. Ochiai, N., Fujimura, M., Oshima, M., Motoyama, T., Ichiishi, A., Yamada-Okabe, H., and Yamaguchi, I. 2002. Effects of iprodione and fludioxonil on glycerol synthesis and hyphal development in Candida albicans. Biosci. Biotechnol. Biochem. 66:2209-2215.

24. Oleastro, M., Menard, A., Santos, A., Lamouliatte, H., Monteiro, L., Barthelemy, P., and Megraud, F. 2003. Real-time PCR assay for rapid and accurate detection of point mutations conferring resistance to clarithromycin in Helicobacter pylori. J. Clin. Microbiol. 41:397-402.

25. Oshima, M., Banno, S., Okada, K., Takeuchi, T., Kimura, M., Ichiishi, A., Yamaguchi, I., and Fujimura, M. 2006. Survey of mutations of a histidine kinase gene $B c O S 1$ in dicarboximide-resistant field isolates of Botrytis cinerea. J. Gen. Plant Pathol. 72:65-73. 
26. Oshima, M., Fujimura, M., Banno, S., Hashimoto, C., Motoyama, T., Ichiishi, A., and Yamaguchi, I. 2002. A point mutation in the two-component histidine kinase $B c O S-1$ gene confers dicarboximide resistance in field isolates of Botrytis cinerea. Phytopathology 92:75-80.

27. Yarden, O., and Katan, T. 1993. Mutations leading to substitutions at amino acids 198 and 200 of beta-tubulin that correlate with benomylresistance phenotypes of field strains of Botrytis cinerea. Phytopathology
83:1478-1483.

28. Yourman, L. F., and Jeffers, S. N. 1999. Resistance to benzimidazole and dicarboximide fungicides in greenhouse isolates of Botrytis cinerea. Plant Dis. 83:569-575.

29. Yourman, L. F., Jeffers, S. N., and Dean, R. A. 2000. Genetic analysis of isolates of Botrytis cinerea sensitive and resistant to benzimidazole and dicarboximide fungicides. Phytopathology 90:851-859. 